ALGORITHMS FOR DIRECT NUMERICAL SIMULATION OF SHEAR-PERIODIC TURBULENCE

U. Schumann

DFVLR Oberpfaffenhofen

Institute of Atmospheric Physics

D-8031 Wessling, Fed. Rep. Germany

Paper presented at the

Ninth International Conference on Numerical Methods in Fluid Dynamics

June $25-29,1984$

CEN, Saclay, France.

Lecture Notes in Physics, Vo1. 218, p. $492-496$.

Springer-Verlag (1985). 


\section{ALGORITHMS FOR DIRECT NUMERICAL SIMULATION OF SHEAR-PERIODIC TURBULENCE}

\section{U. Schumann}

DFVLR Oberpfaffenhofen

Institute of Atmospheric Physics

D-8031 Wessling, Fed. Rep. Germany

\section{Introduction}

This paper describes algorithms for computation of pressure field and for generation of initial conditions as required for direct numerical simulation of homogeneous turbulence generated by vertical shear and buoyancy. The turbulent flow will be simulated by direct numerical integration of the three-dimensional and time-dependent Navier-Stokes equations for viscous and conductive incompressible fluid with constant material properties. Buoyancy is taken into account according to the Boussinesq approximation as induced by a mean vertical temperature gradient. The objective of this work (in prog-ess) is to determine the structure of turbulent shear flow under stable and unstable thermal stratification and to provide data for calibration of second order turbulence closure models.

Direct simulations with e.g. $64^{3}$ grid cells are restricted to moderate Reynolds numbers. Typical values of the Reynolds number based on Taylor-microscale and the root-mean-square velocity are 40 to 60 . In the past, isotropic and axisymmetric homogeneous turbulence have been considered. Such simulations have been successful in providing data for test of turbulence models [1, 2]. In these cases, periodic boundary conditions have been applied in all three coordinate directions.

For the case of shear, periodicity boundary conditions have to be changed with respect to the shear direction. Rogallo $[3,2]$ simulated shear with an Lagrangian approach; he used a computational domain which deforms as a function of time according to the mean hear. In the sheared coordinate system periodic boundary conditions are appropriate. This approach requires remapping of all fields from time to time onto an undeformed domain and this introduces interpolation errors.

An alternative has been proposed by Baron [4]. He uses a fixed=coordinate system but boundary conditions which we call "shear-periodic": If, for example, $f_{B}(x, y, t)$ is a field component at the bottom $(z=0)$ of the computational domain and $f_{T}(x, y, t)$ is the field at the top of the computational domain $(z=H)$, then shear-periodicity means

$$
f_{T}(x, y, t)=f_{B}(x-U t, y, t)+(\partial f / \partial z) H
$$

where $U / H=(\partial u / \partial z)$ is the mean shear of horizontal velocity in $x$-direction. The expression $x-U t$ is taken modulo the horizontal length of periodicity. This type of boundary condition can be implemented easily in a finite difference scheme, see Figure 1. It does not require any interpolation if the time step $\Delta t$ and the grid spacing $\Delta \mathrm{x}$ are taken such that $\mathrm{U} \Delta \mathrm{t} / \Delta \mathrm{x}=1$. 

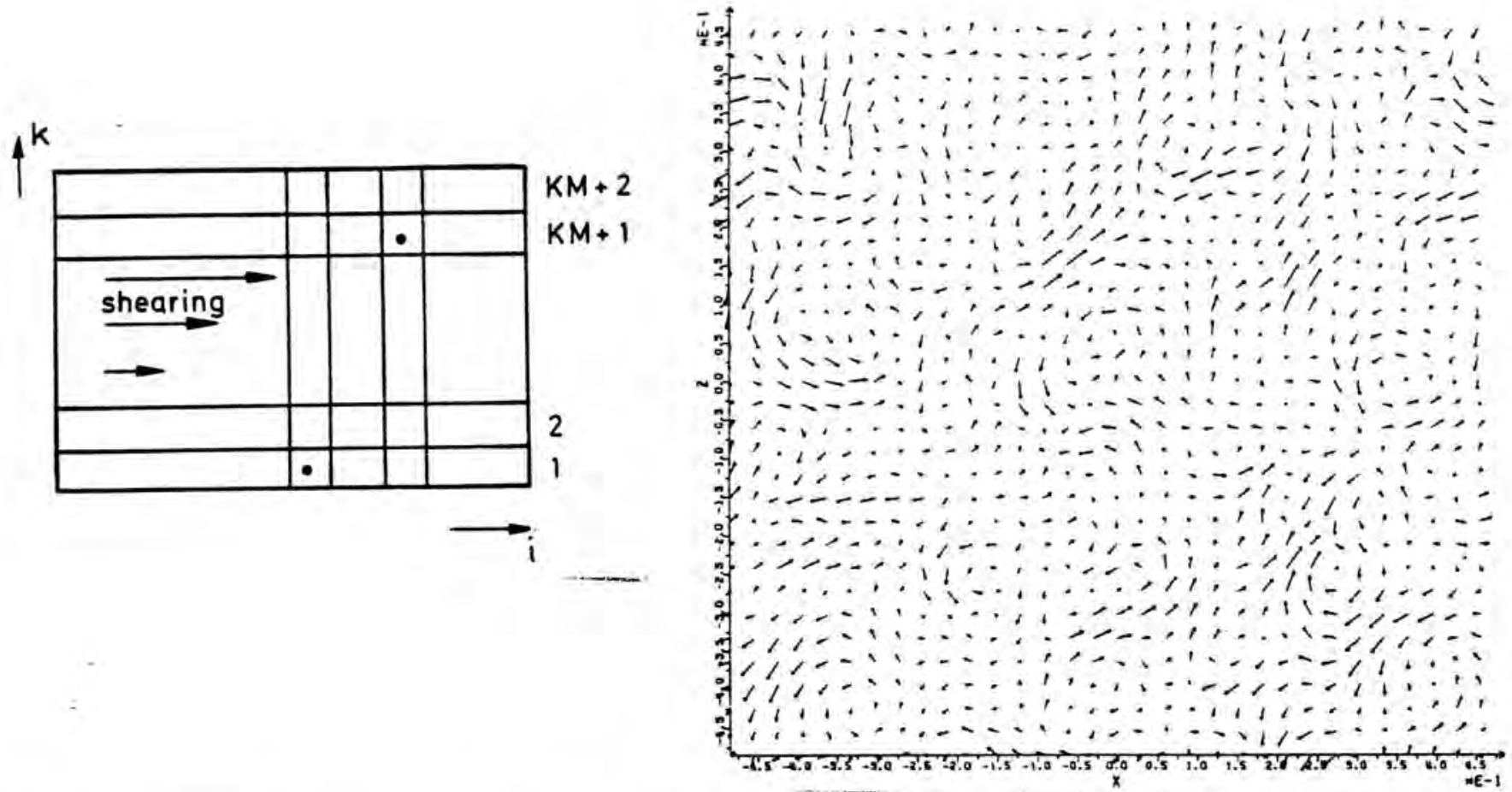

Figure 1. Left: Shear-periodic boundary condition in a finite difference grid. $i$ and $\mathrm{k}$ denote the mesh indices in $\mathrm{x}$ and $\mathrm{z}$ direction; the cells marked with dots get equal values for a given value of $\mu=U t / H . K M \equiv N$.

Right: Velocity field generated for initial conditions; mean field subtracted.

On the other hand, shear periodicity is an unusual boundary condition and no fast Poisson solver - as required to compute the pressure field - is available. Baron used an iterative solution scheme which is very time-consuming. Subsequently, the new Poisson-solver and a new method to generate initial conditions is described briefly.

\section{Direct Poisson-solver for shear-periodicity}

or each time-step, we have to compute the pressure $p(i, j, k)$ from the discrete Poisson equation

$$
\begin{aligned}
& {[p(i-1, j, k)-2 p(i, j, k)+p(i+1, j, k)] / \Delta x^{2}+} \\
& {[p(i, j-1, k)-2 p(i, j, k)+p(i, j+1, k)] / \Delta y^{2}+} \\
& {[p(i, j, k-1)-2 p(i, j, k)+p(i, j, k+1)] / \Delta z^{2}=f(i, j, k),}
\end{aligned}
$$

$i=0,1, \ldots, N-1 ; j=0,1, \ldots N-1 ; k=1,2, \ldots, N ; \Delta x=\Delta y=\Delta z=1 / N$. The boundary conditions are periodic in $\mathrm{i}$ - and $\mathrm{j}$-direction modulo $\mathrm{N}$ and shear-periodic in $\mathrm{k}$-direction. The latter means

$$
p(i, j, 0)=p(i+\mu, j, N), \quad p(i, j, N+1)=p(i-\mu, j, 1)
$$

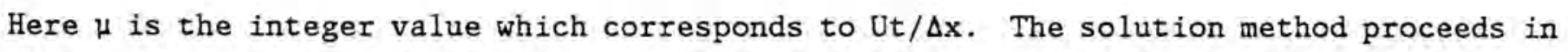
three steps:

First, we determine the complex Fourier-modes and its conjugate pairs 
(3) $f^{*}(m, n, k)=\frac{1}{N \quad N} \sum_{i=0}^{N-1} \sum_{j=0}^{N-1} f(i, j, k) W_{N}^{-m i} W_{N}^{-n j}, f^{*}(N-m, N-n, k)=\left[f^{*}(m, n, k)\right]^{c}$,

(4) $\quad W_{M} \equiv \exp [2 \pi \sqrt{-1} / M]$,

for $m=0,1, \ldots, N / 2 ; n=0,1, \ldots, N-1$, by means of a sequence of two-dimensional FFT's.

Second, with the ansatz

$$
p(i, j, k)=\sum_{m=0}^{N-1} \sum_{n=0}^{N-1} \quad p^{*}(m, n, k) W_{N}^{m i}{ }^{n j}
$$

we obtain a set of one-dimensional linear systems from eq. (1)

$$
\begin{aligned}
& {\left[\mathrm{p}^{*}(\mathrm{~m}, \mathrm{n}, \mathrm{k}-1)-2 \mathrm{p}^{*}(\mathrm{~m}, \mathrm{n}, \mathrm{k})+\mathrm{p}^{*}(\mathrm{~m}, \mathrm{n}, \mathrm{k}+1)\right] / \Delta \mathrm{z}^{2}-\lambda_{\mathrm{m}, \mathrm{n}} \mathrm{p}^{*}(\mathrm{~m}, \mathrm{n}, \mathrm{k})=\mathrm{f}^{*}(\mathrm{~m}, \mathrm{n}, \mathrm{k}),} \\
& \lambda_{\mathrm{m}, \mathrm{n}} \equiv 2[1-\cos (2 \pi \mathrm{m} / \mathrm{N})] / \Delta \mathrm{x}^{2}+2[1-\cos (2 \pi \mathrm{n} / \mathrm{N})] / \Delta \mathrm{y}^{2},
\end{aligned}
$$

$\mathrm{m}=0,1, \ldots, \mathrm{N} / 2, \mathrm{n}=0,1, \ldots, \mathrm{N}-1, \mathrm{k}=1,2, \ldots, \mathrm{N}$, and from eq. (2) the boundary conditions

$$
\begin{aligned}
& \mathrm{p}^{*}(\mathrm{~m}, \mathrm{n}, 0)=\mathrm{W}_{N}^{\mathrm{m}} \mathrm{p}^{*}(\mathrm{~m}, \mathrm{n}, \mathrm{N}), \\
& \mathrm{p}^{*}(\mathrm{~m}, \mathrm{n}, \mathrm{N}+1)=\mathrm{W}_{\mathrm{N}}^{-\mathrm{m} \mu} \mathrm{p}^{*}(\mathrm{~m}, \mathrm{n}, 1) .
\end{aligned}
$$

Subsequently we describe some aspects of the algorithm used to solve eq. (7-8) for each Fourier-mode-index $(m, n)$. For shortness, we omit the indices $m$ and $n$ and define

$$
\begin{aligned}
& \mathrm{p}^{*}(\mathrm{k}) \equiv \mathrm{p}^{*}(\mathrm{~m}, \mathrm{n}, \mathrm{k}) \quad \equiv \mathrm{p}_{\mathrm{R}}(\mathrm{k})+\sqrt{-1} \mathrm{p}_{\mathrm{I}}(\mathrm{k}) \\
& \mathrm{f}^{*}(\mathrm{k}) \equiv \mathrm{f}^{*}(\mathrm{~m}, \mathrm{n}, \mathrm{k}) \Delta \mathrm{z}^{2} \equiv \mathrm{f}_{\mathrm{R}}(\mathrm{k})+\sqrt{ }-1 \mathrm{f}_{I}(\mathrm{k}) \\
& \alpha \quad \equiv-2-\lambda_{\mathrm{m}, \mathrm{n}} \Delta \mathrm{z}^{2} \\
& \mathrm{w}_{\mathrm{N}}^{\mathrm{m \mu}} \equiv \mathrm{w}_{\mathrm{R}}+\sqrt{ }-1 \mathrm{w}_{I}, \mathrm{w}_{\mathrm{R}} \equiv \cos (2 \pi \mathrm{m} \mu / \mathrm{N}), \mathrm{w}_{\mathrm{I}} \equiv \sin (2 \pi \mathrm{m} \mu / \mathrm{N}) .
\end{aligned}
$$

The complex system can be split into real and imaginary parts and ordered such that its band-width becomes minimal. This ordering is essential for effectiveness: 
(9)

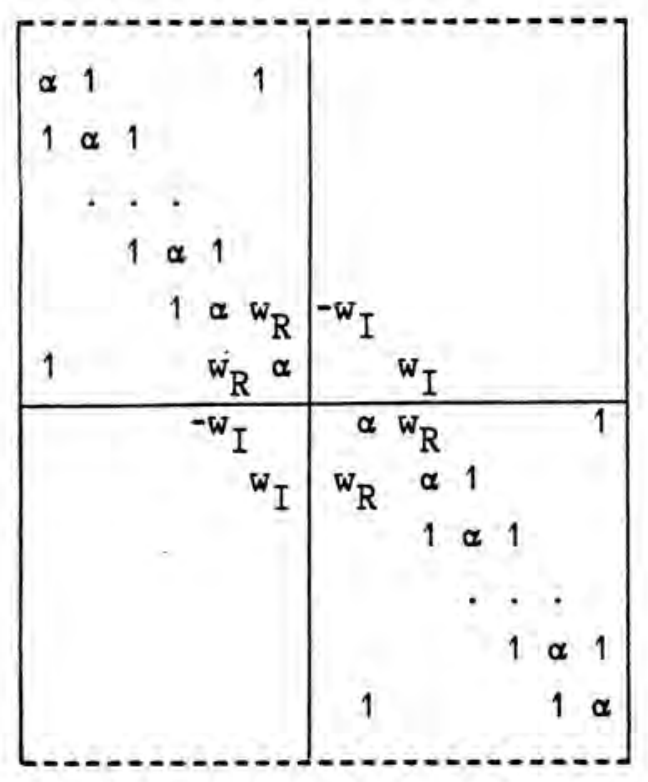

$\left|\begin{array}{l}p_{R}(2) \\ p_{R}(3) \\ \cdot \\ p_{R}(N-1) \\ p_{R}(N) \\ p_{R}(1) \\ \hline p_{I}(1) \\ p_{I}(N) \\ p_{I}(N-1) \\ p_{I}(3) \\ p_{I}(2) \\ -\cdots\end{array}\right|=\left|\begin{array}{l}f_{R}(2) \\ f_{R}(3) \\ f_{R}(N-1) \\ f_{R}(N) \\ f_{R}(1) \\ \hline f_{I}(1) \\ f_{I}(N) \\ f_{I}(N-1) \\ \cdot \\ f_{I}(3) \\ f_{I}(2) \\ -\ldots\end{array}\right|$

his system is solved by Gaussian elimination. The elimination proceeds from the top line downwards and the bottom line upwards up to the equation in the centre. Hereby, the coefficients for the real and imaginary part are equal and this reduces the required computational effort.

For $\mathrm{N} \gg 1$, the number of multiplications is 5.5 and the number of divisions is 0.5 per grid point. This is the same operation-count one would have for the standard periodic case $(m \mu=0)$.

Third, from the solutions $p^{*}(m, n, k)$, we determine the final solution $p(i, j, k)$ according to eq. (5) again by a sequence of two-dimensional FFT's.

The algorithm is fully vectorized by interchanging loops over the indices such that the innermost loops are free of recursions. Typical computing times on a CRAY-1 are $0.36 \mathrm{~s}$ or $\mathrm{N}=64$. Details of the algorithm and a Fortran-subroutine are described in [5].

\section{Generation of initial conditions}

An important issue of all direct numerical simulations is the generation of suitable initial conditions. Here, an algorithm is described which generates random three-dimensional real initial fields for velocity $\left(u_{1}, u_{2}, u_{3}\right)$, and temperatur $T$. The fields are generated such that the velocity field satisfies the discretized continuity equation on a staggered grid and such that the fields follow a prescribed correlation spectrum

$$
\begin{aligned}
R_{i j}(k) & =\sum \Sigma \Sigma f_{i}^{*}(k) f_{j}^{*}(-k) \\
k & \leq|k|<k+1
\end{aligned}
$$

where $k$ is the integer wave-number vector, $\left(f_{1}, f_{2}, f_{3}, f_{4}\right)$ stands for $\left(u_{1}, u_{2}, u_{3}, T\right)$, and the star indicates the complex Fourier mode. This spectrum can be selected arbitrarily except for certain realizability constraints [6]. 
In short, the idea is as follows: By Cholesky-decomposition we determine $\gamma_{i j}$ such that $\gamma_{i n}{ }^{\gamma}{ }_{j}=R^{\prime \prime}{ }_{i j}$ and set $f_{i}^{*}(k)=\gamma_{i j} \alpha_{j}(k)$, where $\alpha_{j}(k)$ are e.g. Gaussian random complex numbers with zero mean and

$\sum \Sigma \Sigma \quad \alpha_{i}(k) \alpha_{j}(-k)=\delta_{i j}$.

$\mathrm{k} \leq|\mathbf{k}|<\mathrm{k}+1$

Thereafter the Fourier modes are corrected to satisfy the Fourier-transformed continuity equation. This correction reduces the degree of anisotropy. The reduction is balanced by setting

$$
\begin{aligned}
& {\left[\begin{array}{l}
R^{\prime \prime} \\
R^{\prime \prime} \\
R^{\prime \prime} \\
33
\end{array}\right]=(1 / 14)\left[\begin{array}{lll}
27 & -3 & -3 \\
-3 & 27 & -3 \\
-3 & -3 & 27
\end{array}\right]\left[\begin{array}{l}
R_{11} \\
R_{22} \\
R_{33}
\end{array}\right],} \\
& \left(R^{\prime \prime}{ }_{12}, R_{13}^{\prime \prime}, R^{\prime \prime}{ }_{23}\right)=(15 / 7)\left(R_{12}, R_{13}, R_{23}\right), \\
& \left(R_{14}^{\prime \prime}, R_{24}^{\prime \prime}, R_{34}^{\prime \prime}\right)=(3 / 2)\left(R_{14}, R_{24}, R_{34}\right), \\
& R_{44}=R_{44}, \quad R_{i j}^{\prime \prime}=R_{j i} .
\end{aligned}
$$

The anisotropy of $R_{i j}$ is limited by the requirement that $R^{\prime \prime}{ }_{i j}$ has to be positive definite. By FFT the real fields are obtained, Details are given in [6].

For example, Figure 1 shows a velocity field generated with this algorithm for $\mathrm{N}=32$.

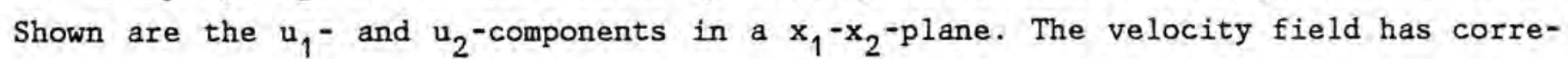
lation spectra of the form $R_{i j}(k) \sim\left(k / k_{p}\right)^{4} \exp \left(-2\left(k / k_{p}\right)^{2}\right)$ with $k_{p}=6, H=1$, and $R_{11}=R_{22}=R_{33}, R_{12}=0.5 R_{11}, R_{13}=R_{23}=0$. The $50 \%$ correlation between $u_{1}$ and $u_{2}$ and the energy-maximum for a wave-length of $\mathrm{H} / 6$ can easily be seen from the figure.

\section{References}

- 1 ] U. Schumann \& G.S.Patterson, Jr.: Numerical Study of Pressure and Velocity Fluctuations in Nearly Isotropic Turbulence. J. Fluid Mech. 88 (1978) 685-709.

[ 2 ] R.M. Kerr: Higher Order Derivative Correlation of Velocity and Temperature in Isotropic and Sheared Numerical Turbulence. 4th Symp. Turbulent Shear Flows, Sept. $12-14,83$, Karls ruhe, 14.9-12.

[ 3 ] R.S. Rogallo: Numerical experiments in homogeneous turbulence. NASA Technical Memorandum 81315 (1981).

[ 4 ] F. Baron: Macro-Simulation Tridimensionelle d'ecoulements turbulents cisailles. These de Docteur-Ingenieur, Univ. Pierre et Marie Curie, Paris 6 (1982).

[ 5 ] H. Schmidt et al.: Three dimensional direct and vectorized elliptic solvers for various boundary conditions. DFVLR-Mitteilung, to appear (1984).

[ 6 ] U. Schumann: Generation of random periodic velocity and temperature fields with prescribed correlation spectra. DFVLR-IB-553-6/84 (1984). 